\title{
Impaired Autoregulation of Glomerular Capillary Hydrostatic Pressure in the Rat Remnant Nephron
}

\author{
Juan C. Pelayo* and Jay Y. Westcott*s \\ Departments of ${ }^{*}$ Pediatrics and ${ }^{\ddagger}$ Cardiovascular Pulmonary Research and ${ }^{\S}$ Webb Waring Lung Institute, \\ University of Colorado School of Medicine, Denver, Colorado 80262
}

\begin{abstract}
In the present micropuncture study, the autoregulation of glomerular capillary hydrostatic pressure $\left(P_{G}\right)$ in Munich-Wistar rats $24 \mathrm{~h}$ after $\mathbf{7 5 \%}$ nephrectomy (Nx) or sham operation (Sh) was investigated. The effect of varying renal perfusion pressure (RPP) on paired determinations of directly measured $\boldsymbol{P}_{G}$ was evaluated in glomeruli of nephrons in which distal fluid delivery was present (unblocked). Autoregulation of $\boldsymbol{P}_{\mathbf{G}}$ in Sh glomeruli with unblocked tubules occurred at RPP values between 99.5 \pm 1.0 and $132.1 \pm 1.0 \mathrm{mmHg}$. In contrast, in $\mathrm{Nx}$ glomeruli with unblocked tubules $P_{G}$ increased by $0.32 \pm 0.07 \mathrm{mmHg}$ / mmHg increase in RPP over this same range of RPP (P $<0.0001)$. To determine whether enhanced prostaglandins synthesis was responsible for the altered regulation of $\boldsymbol{P}_{\mathbf{G}}$ in $\mathbf{N x}$ glomeruli, we repeated the micropuncture measurements in a setting of prostaglandin synthesis inhibition. Although prostaglandins synthesis inhibition did not affect the autoregulation of $\boldsymbol{P}_{\mathrm{G}}$ in Sh glomeruli, it did normalize the autoregulatory capacity for $\boldsymbol{P}_{\mathrm{G}}$ of $\mathrm{Nx}$ glomeruli with unblocked tubules. Thus, acute $\mathrm{Nx}$ is associated with a significant loss of the autoregulatory capacity for $\boldsymbol{P}_{\mathrm{G}}$ and this impairment appears to be related to a prostaglandin-mediated alteration of the responsiveness of the vascular effector site for autoregulation. (J. Clin. Invest. 1991. 88:101-105.) Key words: micropuncture study • nephrectomy • glomerular capillary hydrostatic pressure - renal autoregulation $\bullet$ prostaglandins $\bullet$ cyclooxygenase inhibition
\end{abstract}

\section{Introduction}

Partial nephrectomy $(\mathrm{Nx})^{1}$ in the rat results in hyperfunction and hypertrophy of the remnant nephrons with subsequent development of systemic hypertension, progressive proteinuria, and glomerulosclerosis, and as such has been extensively

Portions of these studies were presented at the 22nd Annual Meeting of the American Society of Nephrology, Washington, DC, 1989, and published in abstract form (1990. Kidney Int. 37:554A).

Address correspondence and reprint requests to Juan C. Pelayo, M.D., UCLA School of Medicine, Division of Pediatric Nephrology, Center for the Health Sciences, A2-331 MDCC, 10833 Le Conte Ave., Los Angeles, CA 90024-1752.

Received for publication 13 April 1990 and in revised form 22 February 1991.

1. Abbreviations used in this paper: CRF, chronic renal failure; Hct, hematocrit; $\mathrm{Nx}$, partial nephrectomy; $P_{\mathrm{G}}$, glomerular capillary hydrostatic pressure; RPP, renal arterial perfusion pressure.

J. Clin. Invest.

(c) The American Society for Clinical Investigation, Inc.

0021-9738/91/07/0101/05 \$2.00

Volume 88, July 1991, 101-105 studied as a model of chronic renal failure (CRF) (1-3). Although the hyperfunctioning state itself appears important to the genesis of nephron injury $(4,5)$, the mechanisms of glomerular and tubular adaptations are not well understood. Recent studies in our laboratory (6) demonstrated that a $45 \%$ reduction in preglomerular vascular resistance entirely accounted for the increase in glomerular capillary hydrostatic pressure $\left(P_{G}\right)$ and in single nephron plasma flow that were demonstrable within $24 \mathrm{~h}$ after Nx . These hemodynamic adjustments to acute $\mathrm{Nx}$ were central to glomerular hyperfiltration and occurred notwithstanding a marked rise in distal tubular fluid flow (6). Based on the tubuloglomerular feedback hypothesis of renal autoregulation which predicts that increased distal tubular fluid flow (or some component thereof) should cause afferent arteriole constriction and therefore a reduction in $P_{\mathrm{G}}(7-9)$, our data suggest that renal autoregulation and in particular the autoregulatory capacity of the remnant nephrons for $P_{\mathrm{G}}$ appears to be either reset or lost after acute Nx. Previous work on renal autoregulation in experimental models of CRF have been limited to investigations at the whole kidney level in the chronic state. These studies have indicated that the autoregulation of glomerular filtration rate and renal blood flow is almost completely absent in rats with CRF secondary to $\mathrm{Nx}(10)$ and glomerulonephritis (11). A preliminary study from our laboratory was compatible with the suggestion that the autoregulation of $P_{\mathrm{G}}$ in chronic Nx is nearly abolished (12). Taken together, these observations indicate that loss of renal autoregulation is a significant feature of CRF and suggest that glomerular capillaries, particularly in $\mathrm{Nx}$, may not be protected from pressure changes in the systemic circulation.

Despite these provocative findings, the mechanism(s) by which Nx results in altered renal autoregulation and the timing of this occurrence remain to be determined. The study presented here was performed to characterize the autoregulation of $P_{\mathrm{G}}$ in remnant nephrons $24 \mathrm{~h}$ after $\mathrm{Nx}$. Our results indicate that acute Nx results in a substantial loss of autoregulation of $P_{\mathrm{G}}$ and that a prostaglandin-dependent alteration of the responsiveness of the vascular effector site (i.e., afferent arteriole) for autoregulation may be critical to this impairment.

\section{Methods}

Experimental animals. Adult male Munich-Wistar rats (Simonsen Laboratories, Inc., Gilroy, CA) were used in this investigation. All animals were given free access to tap water and fed ad libitum a standard pellet diet (Wayne Lab-Blox, Golden K. Feed and Seed, Longmont, CO), containing $\sim 22 \%$ protein by weight.

Surgical preparation for micropuncture studies. Rats were anesthetized with Inactin $\left(100 \mathrm{mg} \cdot \mathrm{kg}\right.$ body $\mathrm{wt}^{-1}$ i.p.; Byk-Gulden-Lomberg, Konstanz, FRG) and placed on a micropuncture table with a servocontrolled heating unit (Yellow Springs Instrument Co., Yellow Springs, $\mathrm{OH}$ ). Body temperature was maintained at $36.5-37.5^{\circ} \mathrm{C}$. A tracheostomy tube (PE-240) was inserted to insure adequate ventila- 
tion. The left external jugular vein was cannulated for the infusion of isotonic $\mathrm{NaCl}-\mathrm{NaHCO}_{3}$, plasma, and vehicle $\left(\mathrm{Na}_{2} \mathrm{CO}_{3}\right)$ or indomethacin (Sigma Chemical Co., St. Louis, MO). An indwelling catheter (PE50) was placed in the left femoral artery to permit the withdrawal of blood samples for the determination of arterial hematocrit (Hct) and to monitor renal arterial perfusion pressure (RPP). RPP was monitored with an electronic pressure transducer model $\mathrm{P} 23 \mathrm{Db}$ and recorded on an amplifier chart recorder (model 8000 S; Gould Inc., Oxnard, CA). An indwelling catheter (PE-50) was also inserted into the bladder for timed urine collections for determination of flow rate $(V)$, and prostaglandin concentration for the calculation of urinary prostaglandin excretion. Urine samples were stored at $-20^{\circ} \mathrm{C}$ until assayed for prostaglandin concentration. The left experimental kidney was surgically exposed via a flank incision and prepared for micropuncture according to standard protocols described previously (13). To compensate for the loss of plasma associated with the surgical preparation required for a micropuncture study, rats received a continuous intravenous infusion

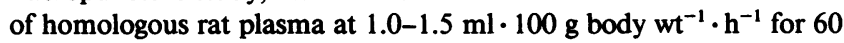
min followed by a maintenance infusion of $0.15-0.2 \mathrm{ml} \cdot 100 \mathrm{~g}$ body $\mathrm{wt}^{-1} \cdot \mathrm{h}^{-1}$ for the duration of each experiment (6). Each sham-operated or nephrectomized rat also received an intravenous infusion of isotonic $\mathrm{NaCl}-\mathrm{NaHCO}_{3}\left(0.5\right.$ or $0.25 \%$ body $\mathrm{wt} \cdot \mathrm{h}^{-1}$, respectively) (6).

Micropuncture measurements of glomerular capillary hydrostatic pressure. Hydrostatic pressure was measured in surface glomerular capillaries and recorded during stepwise reductions of RPP. RPP was varied from $\approx 135$ to $\approx 95 \mathrm{mmHg}$ by $\sim 10 \mathrm{mmHg}$ decrements using an adjustable micrometer screw clamp placed around the abdominal aorta proximal to the origin of the left renal artery (14). To obtain initial RPP at the high end of the desired range, both carotid arteries were occluded at the start of the experiment. This maneuver elevated MAP $\sim 30 \mathrm{mmHg}$ above the baseline level in each animal under investigation. $P_{\mathrm{G}}$ measurements ${ }^{2}$ were directly obtained by a servonulling pressure sensor (Instrumentation for Physiology and Medicine, San Diego, CA) employing long-tapered $0.5-1-\mu \mathrm{m}$ tip diameter glass pipettes filled with $1.2 \mathrm{M} \mathrm{NaCl}(6,15,16)$. Hydrostatic output from the servonull system was monitored with a second electronic pressure transducer and recorded on a second channel of the Gould amplifier chart recorder. The $P_{\mathrm{G}}$ tracings were also displayed on a dual beam oscilloscope (Tektronix Inc., Beaverton, OR) and were compared with the simultaneously displayed femoral artery pressure profiles $(6,16)$. In these experiments, the response of $P_{\mathrm{G}}$ was directly measured in the same glomerulus as RPP was varied and the continued delivery of tubular fluid to the distal nephron segment was not manipulated.

Experimental design. Rats anesthetized with methohexital sodium $\left(50 \mathrm{mg} \cdot \mathrm{kg}\right.$ body $\mathrm{wt}^{-1}$ i.p.; Brevital Sodium, Eli Lilly and Co., Indianapolis, IN) underwent either sham-operation (group Sh, $n=8$ ) or $75 \%$ renal ablation (group $\mathrm{Nx}, n=9$ ) achieved by removal of the right kidney and infarction of approximately half of the left kidney by ligation of two to three branches of the renal artery, as previously described $(6,16)$. The rats were then returned to their individual cages after recovering from anesthesia. Food and water were allowed ad libitum. On the following day, micropuncture measurements of $P_{\mathrm{G}}$ were performed in glomeruli without blockade of distal tubular fluid flow.

The second experimental design consisted also of investigations at one day after either sham operation (group Sh-ID, $n=6$ ) or Nx (group

2. In preliminary studies conducted in our laboratory, it became evident the technical difficulties in maintaining the tip of the pressure pipet in place in a glomerular capillary loop while RPP was modified, because of the changes in kidney volume resulting from this maneuver. To overcome this technical problem, $(a)$ the pressure pipettes were specially designed with a long tapered and small tip to increase their flexibility, $(b)$ the pipettes were inserted into the surface glomeruli at a flat angle $\left(\approx 10-15^{\circ}\right),(c)$ the changes in RPP were effected in a gradual manner, and $(d)$ coincident with these changes in RPP and therefore in kidney volume the position of the pressure pipette was adjusted accordingly by means of a sensitive micromanipulator.
Nx-ID, $n=6$ ) but $P_{\mathrm{G}}$ measurements were obtained during the inhibition of prostaglandin synthesis in glomeruli without blockade of distal tubular flow. Prostaglandin synthesis inhibition was accomplished by the systemic administration of indomethacin $\left(5 \mathrm{mg} \cdot \mathrm{kg}\right.$ body $\mathrm{wt}^{-1}$ i.v., bolus) (6). This cyclooxygenase inhibitor was administered $45 \mathrm{~min}$ before the micropuncture measurements were initiated.

Analytical. Urinary prostaglandin concentrations were quantitated in diluted urine samples by enzyme immunoassay as previously described $(17,18) . \mathrm{PGI}_{2}$ was assessed as its stable metabolite 6-keto$\mathrm{PGF}_{1 \alpha}$ using an antiserum from Advanced Magnetics (Cambridge, MA). This antiserum has a reported cross-reactivity of $8 \%$ with $P \mathrm{FF}_{1 \alpha}$, $7 \%$ with 6-keto-PGE $1,2 \%$ with $\mathrm{PGF}_{2 \alpha}$, and $<1 \%$ with most other prostanoids.

Statistical analysis. All data are expressed as the means \pm SEM. Statistical analysis was performed using a statistics software package (Crunch Software Co., San Francisco, CA) and an IBM Personal System $/ 2$ model 50 computer (IBM Co., Boca Raton, FL). Comparisons between groups were analyzed by unpaired $t$ test. Multiple regression analysis was used to test for equality of two independent slopes. $P$ values $<0.05$ were considered statistically significant.

\section{Results}

There were no significant differences between $\mathrm{Sh}$ and $\mathrm{Nx}$ groups with respect to body weight $(233.1 \pm 5.3$ vs. $234.3 \pm 7.7 \mathrm{~g}$, respectively) and hematocrit $(49.8 \pm 0.4$ vs. $50.7 \pm 0.6$ vol\%, respectively). Similarly, the mean values for body weight and hematocrit were not different between Sh-ID and Nx-ID groups $(276.0 \pm 11.6$ vs. $278.2 \pm 12.4 \mathrm{~g}$ and $49.7 \pm 1.0$ vs. $49.1 \pm 0.6$ vol\%, respectively).

Relationship of $P_{G}$ to RPP in Sh and Nx groups. Fig. 1 depicts the $P_{\mathrm{G}}$ responses to changes in RPP in Sh glomeruli (group $\mathrm{Sh}$ ) without interruption of distal fluid delivery. Autoregulation of $P_{\mathrm{G}}$ was documented at levels of RPP ranging from $99.5 \pm 1.0$ to $132.1 \pm 1.0 \mathrm{mmHg}$. The slope of this relationship demonstrated that $P_{\mathrm{G}}$ changed only by $0.05 \pm 0.05 \mathrm{mmHg} /$ mmHg variation in RPP $(r=0.18, P>0.3)$. However, when RPP was either higher or lower than that range, $P_{G}$ was found to be highly RPP dependent.

Having established in Sh glomeruli without interruption of distal fluid delivery, the range of RPP in which $P_{\mathrm{G}}$ is autoregulated under euvolemic conditions in our laboratory, the responses of $P_{G}$ to changes in RPP in the remaining experimental conditions were assessed within this range of RPP. Fig. 1 shows

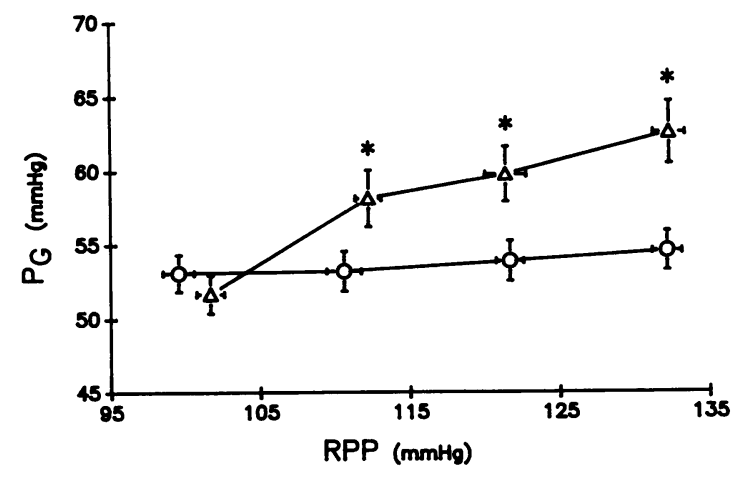

Figure 1. Effect of varying renal perfusion pressure (RPP) on glomerular capillary hydrostatic pressure $\left(P_{G}\right)$ in Sh glomeruli $(0)$ without interruption of distal fluid delivery. Comparison of the responses of $P_{\mathrm{G}}$ to changes in RPP between Sh and Nx $(\Delta)$ glomeruli without interruption of distal fluid delivery. Values plotted are the means \pm SEM. ${ }^{*} P<0.05 \mathrm{Nx}$ vs. Sh. 
that in Nx glomeruli (group Nx) without interruption of distal fluid delivery a relatively steep relationship existed between $P_{\mathrm{G}}$ and RPP. Analysis of the slope of this relationship demonstrated that a mmHg increase in RPP resulted in an increase in $P_{\mathrm{G}}$ of $0.32 \pm 0.07 \mathrm{mmHg}(r=0.6, P<0.0001)$. Mean values of $P_{\mathrm{G}}$ in group Nx compared with those in group Sh at RPP of $112.0 \pm 0.8$ vs. $110.6 \pm 1.2,121.4 \pm 1.4$ vs. $121.7 \pm 0.9$, and $132.2 \pm 0.8$ vs. $132.1 \pm 1.0 \mathrm{mmHg}$, respectively, were significantly higher averaging $58.1 \pm 1.9$ vs. $53.2 \pm 1.3, P<0.05$, $60.0 \pm 1.8$ vs. $53.9 \pm 1.4, P<0.025$, and $62.6 \pm 2.1$ vs. $54.6 \pm 1.3$ $\mathrm{mmHg}, P<0.005$, respectively. It should be noticed that the slope of the normalized responses of $P_{\mathrm{G}}$ to changes in RPP in Nx glomeruli without blocked proximal tubules was significantly less than the unity slope $(P<0.05)$. This line of identity, in which the coordinates were expressed on a relative scale (i.e., percent changes), represents the theoretical response of $P_{\mathrm{G}}$ to changes in RPP in the absence of autoregulatory resistances changes in the renal microcirculation (19). Thus, acute $\mathrm{Nx}$ resulted in higher values of $P_{\mathrm{G}}$ and in a significant loss of the capacity for autoregulation of $P_{\mathrm{G}}$.

Effect of prostaglandin synthesis inhibition on the relationship of $P_{G}$ to RPP in Sh-ID and Nx-ID groups. The autoregulatory capacity for $P_{\mathrm{G}}$ in Sh-ID glomeruli with intact distal fluid delivery was not altered by the inhibition of prostaglandin synthesis (group Sh-ID, Fig. 2). $P_{\mathrm{G}}$ changed on average by $0.06 \pm 0.05 \mathrm{mmHg} / \mathrm{mmHg}$ alteration in RPP $(r=0.18, P$ $>0.2$ ).

Fig. 2 also presents the effect of prostaglandin synthesis inhibition on the relationship between $P_{\mathrm{G}}$ and RPP in Nx-ID glomeruli without interruption of distal fluid delivery (group $\mathrm{Nx}$-ID). Important to the interpretation of this study was the finding that prostaglandin synthesis inhibition was associated with a normal autoregulatory behavior in Nx-ID glomeruli with maintenance of distal fluid delivery. Analysis of the relationship between RPP and $P_{\mathrm{G}}$ indicated that $P_{\mathrm{G}}$ varied only by $0.09 \pm 0.05 \mathrm{mmHg} / \mathrm{mmHg}$ change in RPP $(r=0.2, P>0.1)$. This slope was not different from that obtained in Sh-ID $(P$ $>0.3$ ). Similarly, the mean values for $P_{\mathrm{G}}$ at the various levels of RPP measured were not different between groups.

Urinary prostaglandin excretion. As depicted in Table I, absolute urinary excretion of 6-keto-PGF ${ }_{1 \alpha}$ in group Sh was significantly higher than in group $\mathrm{Nx}$. However, correction of the absolute urinary excretion rate of 6-keto- $\mathrm{PGF}_{1 \alpha}$ by kidney mass revealed a twofold increase in the excretory rate of this

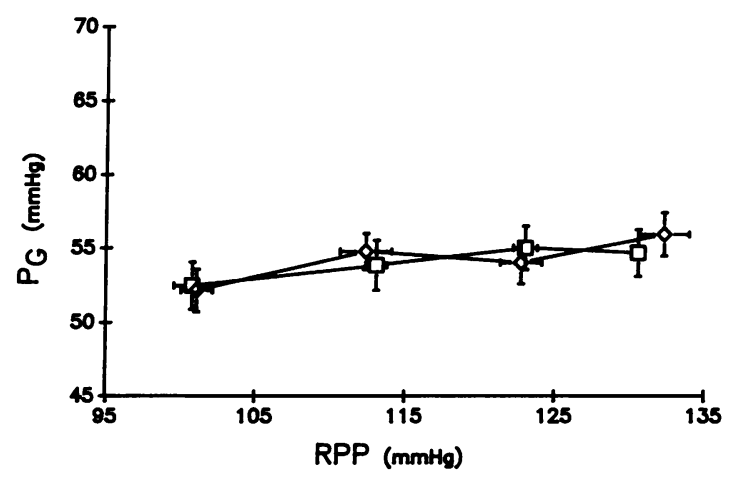

Figure 2. Effect of prostaglandin synthesis inhibition on the relationship of $P_{\mathrm{G}}$ to RPP in Sh-ID (ㅁ) and Nx-ID $(\diamond)$ glomeruli without interruption of distal fluid delivery.
Table I. Urinary Prostaglandin Concentration and Excretion Rate, and Urine Flow Rate in Groups Sh, Sh-ID, Nx, and Nx-ID

\begin{tabular}{lccr}
\hline \multicolumn{3}{c}{ 6-keto-PGF Ia } & \multicolumn{1}{c}{$V$} \\
\hline & $n g / m l$ & $p g /$ min & $\mu l / m i n$ \\
Group & & & \\
Sh $(n=8)$ & $23.8 \pm 2.8$ & $231.6 \pm 29.5$ & $11.5 \pm 2.6$ \\
Sh-ID $(n=6)$ & $22.5 \pm 9.5$ & $69.9 \pm 16.5^{*}$ & $5.4 \pm 2.7$ \\
Nx $(n=9)$ & $11.0 \pm 1.9^{\ddagger}$ & $129.6 \pm 14.7^{\ddagger}$ & $13.4 \pm 1.6$ \\
Nx-ID $(n=6)$ & $8.1 \pm 1.7$ & $44.3 \pm 15.4^{\S}$ & $6.5 \pm 2.6^{8}$ \\
& & & \\
\hline
\end{tabular}

Values are means \pm SE. $n$, No. of rats. ${ }^{*} P<0.05$ group Sh vs. group Sh-ID. ${ }^{\ddagger} P<0.05$ group Sh vs. group NS. ${ }^{\delta} P<0.05$ group Nx vs. group Nx-ID.

prostaglandin in group $\mathrm{Nx}$ in comparison with group Sh. After pretreatment with indomethacin, the baseline values for the absolute excretory rates of 6-keto-PGF ${ }_{1 \alpha}$ in Sh-ID and Nx-ID groups were markedly and similarly reduced when compared with those in Sh and Nx groups, respectively. Thus, these results are consistent with a significant and similar degree of inhibition of renal $\mathrm{PGI}_{2}$ synthesis in remnant (group $\mathrm{Nx}$-ID) and intact (group Sh-ID) kidneys by the acute administration of indomethacin.

\section{Discussion}

The topic of renal autoregulation in experimental models of CRF has been previously studied at the whole organ level in the chronic state of $\mathrm{Nx}(10)$ and glomerulonephritis (11). On the basis of these results as well as data attained in our recent micropuncture investigations in both acute (6) and chronic (12) $\mathrm{Nx}$, it can be inferred that the capacity for renal autoregulation is significantly impaired in experimental models of CRF associated with significant nephron loss. Still unanswered are the questions of whether loss of renal autoregulation, particularly for $P_{\mathrm{G}}$, occurs in the acute state of $\mathrm{Nx}$, and if so, what are the pathogenetic mechanisms responsible for this derangement. The unique features of the present investigation were that autoregulation of $P_{\mathrm{G}}$ was directly assessed by micropuncture techniques in the same glomerulus at several different levels of RPP and that the study was designed to ascertain the contribution of potential mediators, namely prostaglandin. The major findings were $(a)$ a substantial loss of autoregulatory capacity for $P_{\mathrm{G}}$ occurred in remnant nephrons $24 \mathrm{~h}$ after $\mathrm{Nx}$, and $(b)$ cyclooxygenase inhibition studies suggest a pathogenetic mechanism dependent on vasodilatory prostaglandin.

Theoretical concepts on renal autoregulation have suggested at least two putative mechanisms, the tubuloglomerular feedback and the myogenic $(20,21)$. In fact, several studies have provided evidence suggesting the importance of the tubuloglomerular feedback mechanism in regulating glomerular capillary hydrostatic pressure responses to changes in $\operatorname{RPP}(19$, 22-25). These important investigations have conclusively demonstrated that stop-flow pressure, an indirect index of directly measured $P_{\mathrm{G}}$, varies directly with RPP when distal fluid delivery is zero, even when measured at RPP levels within the range of autoregulation $(19,22-24)$. On the other hand, in microperfusion studies (24) in which distal fluid delivery was main- 
tained at a high rate, stop-flow pressure was independent of changes in RPP. The results are in agreement with an earlier study that had directly measured $P_{\mathrm{G}}$ responses to changes in RPP and had demonstrated autoregulation of $P_{\mathrm{G}}$ in glomeruli from nephrons without interruption of distal fluid delivery (25). Thus, the present experiments duplicate the previously described studies, demonstrating that directly measured $P_{\mathrm{G}}$ in Sh glomeruli from unblocked nephrons was autoregulated within the predictable range of RPP.

In contrast to Sh glomeruli, $\mathrm{Nx}$ glomeruli in which distal fluid delivery was not interrupted, did not autoregulate $P_{\mathrm{G}}$. Furthermore, in the Nx group the slope of the relationship between $P_{\mathrm{G}}$ and RPP was significantly less than the unity slope, suggesting the presence, albeit small, of residual autoregulatory capacity in remnant glomeruli. Taken together, these results suggest that in the pathological condition of acute nephron loss, the autoregulatory capacity for $P_{\mathrm{G}}$ is significantly impaired in Nx glomeruli. The defect or defects that may explain the substantial loss of autoregulation of $P_{\mathrm{G}}$ in this model are presently unknown and might reside in the myogenic mechanism (21) and/or in a single component or a combination of the three components of the tubuloglomerular feedback mechanism (i.e., signal, sensor, and effector sites) (7-9). Although the findings presented above do not permit a determination as to which component(s) is altered, based on our previous study in which we documented that a $45 \%$ reduction in afferent arteriolar resistance was the mechanism to account entirely for the observed glomerular hypertension and hyperperfusion, and therefore glomerular hyperfiltration $24 \mathrm{~h}$ after $\mathrm{Nx}$ (6), one could speculate that a lack of responsiveness at this afferent vascular effector site may be the critical alteration. It is important to mention that several lines of evidence have pointed to the afferent arteriole as the primary vascular site for the interaction of myogenic and tubuloglomerular feedback mechanisms, and renal autoregulation (25-27). The possibility that an altered tubuloglomerular feedback mechanism might contribute to the impairment of $P_{\mathrm{G}}$ autoregulation in the experimental model of nephron loss is supported by a previous study which suggested a decreased sensitivity of the tubuloglomerular feedback mechanism $1 \mathrm{~h}$ after uninephrectomy (15).

The present data obtained from the cyclooxygenase inhibition experiments are especially significant when viewed in the context of our previous investigation (6) and together they provide a clue as to which component (i.e., vascular effector site) may be altered in acute Nx. In this rat model of CRF, we have recently demonstrated in the acute state that the urinary excretory rate per nephron for $\mathrm{PGI}_{2}$ and $\mathrm{Tx} \mathrm{A}_{2}$, a vasodilatory and a vasoconstrictor prostaglandin, respectively, are significantly increased (6). Prostaglandin synthesis inhibition resulted in normalization of glomerular hyperfiltration, hyperperfusion, and hypertension by preventing dilation of the afferent vascular site in remnant nephrons $24 \mathrm{~h}$ after $\mathrm{Nx}$ (6). These data strongly suggested that a vasodilatory prostaglandin, conceivably $\mathrm{PGI}_{2}$, dilates preglomerular vascular sites in remnant nephrons, overcoming any potential effect of vasoconstrictor hormones including the prostaglandin $\mathrm{TxA}_{2}$.

In the present study prostaglandin synthesis inhibition was confirmed by quantitation of 6-keto-PGF ${ }_{1 \alpha}$ excretion. The autoregulation of $P_{\mathrm{G}}$ in Sh-ID glomeruli from unblocked nephrons was not modified. However, under the same condition the autoregulatory capacity for $P_{\mathrm{G}}$ in $\mathrm{Nx}$-ID glomeruli without interruption of distal fluid delivery was normalized.
This observation is most consistent with a pathogenetic mechanism that involves the effect(s) of a prostaglandin. This finding, in conjunction with our previous report (6), suggests that a vasodilatory prostaglandin, possibly $\mathrm{PGI}_{2}$, whose synthesis is increased in acute $\mathrm{Nx}$ dilates the afferent arteriole of remnant nephrons rendering this vascular effector site significantly unresponsive to changes in the stimulus provided by autoregulatory mechanisms, namely, tubuloglomerular feedback and myogenic. Of interest, indomethacin administration to rats with unilateral nephrectomy restored the sensitivity of the tubuloglomerular feedback mechanism in transplanted kidneys (32). This interpretation does not preclude the possibility that other factor(s) could also play a role in the documented derangement of $P_{\mathrm{G}}$ autoregulation in remnant nephrons (28).

In this regard, it is of interest to consider the relationship of renal oncotic and hydrostatic interstitial pressures to tubuloglomerular feedback mechanism activity (29). It has been argued on the basis of a large body of evidence that there is an inverse relationship between net renal interstitial pressure (i.e., the difference between the interstitial hydrostatic and oncotic pressures) and tubuloglomerular feedback mechanism activity $(29,30)$. We have previously demonstrated an increase in net renal interstitial pressure in remnant kidneys after acute $\mathrm{Nx}$ (6). This augmentation was primarily due to a marked increase in interstitial hydrostatic pressure. In addition, prostaglandin synthesis inhibition was associated with normalization of renal interstitial hydrostatic pressure (6). Thus, there is reasonable evidence to hypothesize that the increase in net renal interstitial pressure in remnant kidneys after acute $\mathrm{Nx}$, an alteration which appears to be linked to enhanced prostaglandin production, may well contribute to the significant loss of autoregulation of $P_{\mathrm{G}}$ in remnant nephrons.

In summary, the current study documents for the first time with direct micropuncture techniques that the autoregulation of $P_{\mathrm{G}}$ is markedly lost after acute Nx. It seems likely that a prostaglandin-related alteration of the vascular effector site may be an important pathophysiological mechanism in this perturbation. In teleological terms, this derangement in the autoregulatory capacity for $P_{\mathrm{G}}$ in remnant nephrons contributes to the maintenance of solute and water balance in acute $\mathrm{Nx}$ by increasing single nephron glomerular filtration rate (i.e., filtered load) and thus augmenting the excretion of fluid by each remaining nephron (6). In experimental models of CRF it has been hypothesized that glomerular hypertension leads to glomerular injury and eventually to glomerulosclerosis and that this contributes to the progression of renal disease (3-5, $10,31)$. Moreover, the detrimental effect of systemic arterial hypertension on the progression of renal disease has been recognized in both experimental and human renal diseases (31). Thus, the current investigation highlights the importance of an altered autoregulatory behavior of remnant nephrons and provides a mechanism by which systemic arterial hypertension may hasten the progression of renal disease.

\section{Acknowledgments}

The authors gratefully acknowledge the advice provided by Dr. T. J. Burke and Dr. L. G. Navar in preparing this manuscript. The authors thank Almalinda Gonzalez for her technical assistance.

This study was supported by National Institutes of Health Grants BRSG-05357, DK-37706, and HL-34303, and a Grant-in-Aid from the American Heart Association (Colorado Affiliate). 


\section{References}

1. Chanutin, A., and E. B. Ferris. 1932. Experimental renal insufficiency produced by partial nephrectomy. I. Control diet. Arch. Intern. Med. 49:767-787.

2. Shimamura, T., and A. B. Morrison. 1975. A progressive glomerulosclerosis occurring in partial five-sixths nephrectomized rats. Am. J. Pathol. 79:95-106.

3. Hostetter, T. H., J. L. Olson, H. G. Rennke, M. A. Venkatachalam, and B. M. Brenner. 1981. Hyperfiltration in remnant nephrons: a potentially adverse response to renal ablation. Am. J. Physiol. 241(Renal Fluid and Electrolyte Physiol. 10):F85-F93.

4. Brenner, B. M. 1985. Nephron adaptation to renal injury or ablation. Am J. Physiol. 249(Renal Fluid and Electrolyte Physiol. 18):F324-F337.

5. Klahr, S., G. Schreiner, and I. Ichikawa. 1988. The progression of renal disease. N. Engl. J. Med. 318:1657-1666.

6. Pelayo, J. C., and P. F. Shanley. 1990. Glomerular and tubular adaptive responses to acute nephron loss in the rat: effect of prostaglandin synthesis inhibition. J. Clin. Invest. 85:1761-1769.

7. Navar, L. G., D. W. Ploth, and P. D. Bell. 1980. Distal tubular feedback control of renal hemodynamics and autoregulation. Annu. Rev. Physiol. 42:557572.

8. Blantz, R. C., and J. C. Pelayo. 1984. Functional role for the tubuloglomerular feedback mechanism. Kidney Int. 25:739-746.

9. Briggs, J. P., and J. Schnermann. 1987. The tubuloglomerular feedback mechanism: functional and biochemical aspects. Annu. Rev. Physiol. 49:251273.

10. Bidani, A. K., M. M. Schwartz, and E. J. Lewis. 1987. Renal autoregulation and vulnerability to hypertensive injury in remnant kidney. Am. J. Physiol. 252(Renal Fluid and Electrolyte Physiol. 21):F1003-F1010.

11. Iversen, B. M., and J. Ofstad. 1988. Loss of renal blood flow autoregulation in chronic glomerulonephritic rats. Am. J. Physiol. 254(Renal Fluid and Electrolyte Physiol. 23):F284-F290.

12. Pelayo, J. C., and A. G. Quan. 1989. Pathophysiology of glomerular hemodynamic adaptations to nephron loss. Semin. Nephron. 9:10-13.

13. Pelayo, J. C., M. G. Ziegler, P. A. Jose, and R. C. Blantz. 1983. Renal denervation in the rat: analysis of glomerular and proximal tubular function. $\mathrm{Am}$. J. Physiol. 244(Renal Fluid and Electrolyte Physiol. 13):F70-F77.

14. Pelayo, J. C., R. D. Fildes, G. M. Eisner, and P. A. Jose. 1983. Effects of dopamine blockade on renal sodium excretion. Am. J. Physiol. 245(Renal Fluid and Electrolyte Physiol. 14):F247-F253.

15. Muller-Suur, R., B. J. Norlen, and E. G. Persson. 1980. Resetting of tubuloglomerular feedback in rat kidneys after unilateral nephrectomy. Kidney Int. 18:48-57.

16. Pelayo, J. C., A. H. Quan, and P. F. Shanley. 1990. Angiotensin II control of the renal microcirculation in rats with reduced renal mass. Am. J. Physiol. 258(Renal Fluid and Electrolyte Physiol. 27):F414-F422.
17. Pradelles, P., J. Grassi, and J. Maclouf. 1985. Enzyme immunoassays of eicosanoids using acetylcholine esterase as label: an alternative to radioimmunoassay. Anal. Chem. 57:1170-1173.

18. Wenzel, S. E., J. Y. Westcott, H. R. Smith, and G. L. Larsen. 1989. Spectrum of prostanoid release after bronchoalveolar allergen challenge in atopic asthmatics and in control groups. Am. Rev. Respir. Dis. 139:450-457.

19. Moore, L. C. 1984. Tubuloglomerular feedback and SNGFR autoregulation in the rat. Am. J. Physiol. 247(Renal Fluid and Electrolyte Physiol. 16):F267-F276

20. Navar, L. G. 1978. Renal autoregulation: perspectives from whole kidney and single nephron studies. Am. J. Physiol. 234(Renal Fluid and Electrolyte Physiol. 3):F357-F370.

21. Aukland, K., and A. H. Oien. 1987. Renal autoregulation: models combining tubuloglomerular feedback and myogenic response. Am. J. Physiol. 252(Renal Fluid and Electrolyte Physiol. 21):F768-F783.

22. Navar, L. G., B. Chomdej, and P. D. Bell. 1975. Absence of estimated glomerular pressure autoregulation during interrupted distal delivery. Am. J. Physiol. 229:1596-1603.

23. Bell, P. D., and L. G. Navar. 1979. Stop-flow pressure feedback responses during reduced renal vascular resistance in the dog. Am. J. Physiol. 237(Renal Fluid and Electrolyte Physiol. 6):F204-F209.

24. Schnermann, J., and J. P. Briggs. 1989. Interaction between loop of Henle flow and arterial pressure as determinants of glomerular pressure. Am. J. Physiol. 256(Renal Fluid and Electrolyte Physiol. 25):F421-F429.

25. Robertson, C. R., W. M. Deen, J. L. Troy, and B. M. Brenner. 1972. Dynamics of glomerular ultrafiltration in the rat. III. Hemodynamics and autoregulation. Am. J. Physiol. 223:1191-1200.

26. Briggs, J. P., and F. S. Wright. 1979. Feedback control of glomerular filtration rate: site of the effector mechanism. Am. J. Physiol. 236(Renal Fluid and Electrolyte Physiol. 5):F40-F47.

27. Carmines, P. K., E. W. Inscho, and R. C. Gensure. 1990. Arterial pressure effects on preglomerular microvasculature of juxtamedullary nephrons. Am. J. Physiol. 258(Renal Fluid and Electrolyte Physiol. 27):F94-F102.

28. Bell, P. D., M. Franco, and L. G. Navar. 1987. Calcium as a mediator of tubuloglomerular feedback. Annu. Rev. Physiol. 49:275-293.

29. Arendshorst, W. J. 1987. Altered reactivity of tubuloglomerular feedback. Annu. Rev. Physiol. 49:295-317.

30. Selen, G., and A. E. G. Persson. 1983. Hydrostatic and oncotic pressures in the interstitium of dehydrated and volume expanded rats. Acta Physiol. Scand. 117:75-81.

31. Baldwin, D. S., and J. Neugarten. 1986. Blood pressure and progression of renal insufficiency. In The Progressive Nature of Renal Disease. W. E. Mitch, B. M. Brenner, and J. H. Stein, editors. Churchill Livingstone/New York. 81110 